EXTENDED REPORT

\title{
Augmented trabeculectomy in paediatric glaucoma
}

\author{
R Ehrlich, M Snir, M Lusky, D Weinberger, R Friling, D D Gaton
}

Br J Ophthalmol 2005;89:165-168. doi: 10.1136/bjo.2004.046037

See end of article for authors' affiliations

Correspondence to: $R$ Ehrlich, MD, Department of Ophthalmology, Rabin Medical Center, Beilinson Campus, Petah Tiqva 49 100, Israel; ehrlichy@ netvision.net.il

Accepted for publication 12 July 2004

\begin{abstract}
Aims: To report the experience with trabeculectomy augmented with mitomycin $\mathrm{C}$ and 5 -fluorouracil for the treatment of paediatric glaucoma.

Methods: Retrospective, interventional case series design was used. The sample included 17 children (29 eyes) with primary (19 eyes) or secondary (10 eyes) glaucoma who were treated with augmented trabeculectomy as the primary procedure between 1990 and 2002. Data were collected on age and family history, preoperative and end of follow up intraocular pressure, cup/disc ratio (evaluated by drawing), visual acuity, complications, and post-surgery treatment.

Results: Patient age at surgery ranged from 1 month to 8 years; most patients $(n=14,82.3 \%)$ were aged less than 1 year (range 1 month-8 months, mean 3.95 (SD 2.56) months); three patients (17.7\%) were aged 3, 5, and 8 years. The duration of follow up was 3-120 months (mean 46 months). Intraocular pressure significantly improved from $21 \mathrm{~mm} \mathrm{Hg}$ to $60 \mathrm{~mm} \mathrm{Hg}$ (mean 33.1 (10) mm Hg) before surgery to 6-26 mm Hg (mean $17.1(6) \mathrm{mm} \mathrm{Hg}$ ) after, $(\mathrm{p}<0.0001)$. There was no significant change in cup/disc ratio: $0.1-0.8$ (mean $0.42(0.26))$ before and $0.1-1.0$ (mean $0.511(0.27))$ after $(p=0.45)$. In 22 eyes (75.8\%), intraocular pressure was controlled at less than $20 \mathrm{~mm} \mathrm{Hg}$ and the cup/disc ratio remained stable or improved. The life table success rate for intraocular pressure control remained stable at $86 \%$ at the 12, 24, and 36 months and after 48 months decreased to $53 \%$. There was no significant difference in the life table results between primary and secondary glaucoma. 14 eyes $(48.2 \%)$ had a visual acuity better than $20 / 120$ by the end of follow up. Repeated surgery was necessary in eight eyes $(27.5 \%)$, and additional antiglaucoma treatment in $13(44.8 \%)$. Complications included retinal detachment 1 year after surgery, choroidal detachment, and blebitis (one eye each).

Conclusions: Augmented trabeculectomy with mitomycin $C$ and 5 -fluorouracil may serve as the primary procedure in a selected group of paediatric patients with glaucoma.
\end{abstract}

ongenital glaucoma is a severe, potentially blinding disease. ${ }^{1}$ Surgery is the treatment of choice, ${ }^{2}$ with options including goniotomy, trabeculotomy, and trabeculectomy. ${ }^{23}$ Trabeculectomy augmented with antimetabolites is used mostly in cases of unclear media or abnormalities of the angle that preclude goniotomy or trabeculotomy, or when the operating surgeon has insufficient mastery of the other procedures. ${ }^{23}$

The aim of this study was to report the experience of a major tertiary care medical centre with trabeculectomy combined with mitomycin C (MMC) and 5-fluorouracil (5-FU) as the first surgical intervention for paediatric glaucoma.

\section{PATIENTS AND METHODS}

The patient files were reviewed for all children with primary or secondary glaucoma who underwent augmented trabeculectomy as the primary procedure at Schneider Children's Medical Center of Israel from 1990 to 2002. Seventeen children (29 eyes) were identified; all were operated on by the same surgeon (ML) and meticulously followed by a paediatric ophthalmologist (MS).

Data were collected on age at diagnosis and surgery, birth weight, family history, before and end of follow up visual acuity by age, cycloplegic refraction (with cyclopentolate $0.5 \%$, two instillations a half hour apart), intraocular pressure (IOP), cup/disc ratio (evaluated by drawing), corneal diameter, and surgical complications.

Follow up was conducted 1 day, 1 week, 1 month, 3 months, 6 months, and 12 months after surgery, and thereafter every 6 months. Patients who needed closer follow up, according to the clinical outcome, were evaluated monthly or even weekly. Ocular and medical problems, reoperation, and use of antiglaucoma medications after surgery were assessed.
The reasons for treatment with augmented trabeculectomy with antimetabolites over goniotomy or trabeculetomy were presence of severe corneal oedema, severe anatomical malformation that prevented the exposure of Schlemm's canal, and preference and experience of the surgeon. In all patients except one, who had been previously operated on in another hospital, augmented trabeculectomy was the primary procedure, performed in an area of intact conjunctiva. Patients with primary glaucoma were operated early, within a month after diagnosis. Until surgery was performed, they were treated with topical antiglaucoma medications. Patients with secondary glaucoma were treated first with maximal antiglaucoma treatment, and surgery was performed after pharmacological mean failed.

\section{Surgical procedure}

The sclera was exposed at the upper nasal limbal zone. A sponge soaked with $0.2-0.4 \mathrm{mg} / \mathrm{ml} \mathrm{MMC}$ was applied at the filtering site on the upper nasal limbus for 3 minutes, and the area was washed out with a balanced salt solution. A fornix based conjunctival flap was formed in the superior nasal quadrant, followed by a $4 \times 4 \mathrm{~mm}$ triangular partial thickness scleral flap, which was prepared by dissecting the sclera forward into the clear cornea. An internal section of the trabecular meshwork was removed with a Kelly punch, and a small peripheral iridectomy was performed in the same region. The scleral flap was closed with one 10/0 nylon suture, placed at the apex. The conjunctival wound was closed with two 8/0 Vicryl sutures. At the end of surgery, $5 \mathrm{mg}$ of 5 -FU were injected subconjunctivally, $90^{\circ}$ distal to or above the filter zone.

Abbreviations: 5-FU, 5-fluorouracil; MMC, mitomycin C 


\begin{tabular}{|c|c|}
\hline \multicolumn{2}{|l|}{ Sex } \\
\hline $\begin{array}{l}\text { Male } \\
\text { Female }\end{array}$ & $\begin{array}{l}12 \text { patients }(70.6 \%) \\
5 \text { patients }(29.4 \%)\end{array}$ \\
\hline \multicolumn{2}{|l|}{ Age } \\
\hline$<1$ year (3-8 months) & 14 patients (82.3\%) \\
\hline$>1$ year ( $3-8$ years) & 3 patients ( $17.7 \%)$ \\
\hline \multicolumn{2}{|l|}{ Follow up } \\
\hline Range & $3-120$ months \\
\hline Mean (SD) & $46.32(32.88)$ months \\
\hline
\end{tabular}

Surgical success was defined as stabilisation or improvement in the optic disc appearance and IOP below $20 \mathrm{~mm} \mathrm{Hg}$.

\section{Statistical analysis}

Paired samples $t$ test was used for the preoperative and postoperative (end of follow up) comparisons of parameters; p $<0.05$ was considered statistically significant. KaplanMeier survival analysis was also performed. All analyses were done with SPSS software (version 10, Professional Statistics Release, Chicago, IL, USA).

\section{RESULTS}

The demographic data are presented in table 1 . The study group consisted of 12 male $(70.6 \%)$ and five female $(29.4 \%)$ patients. Most patients $(\mathrm{n}=14,82.3 \%)$ were aged less than 1 year (range 1-8 months, mean 3.95 (SD 2.56) months); three patients $(17.7 \%)$ were aged 3,5 , and 8 years. All children were white. All were born at term after a normal pregnancy; birth weight ranged from 2540-4850 g (mean 3334 (624.4) g). The diagnoses are shown in table 2. In three of the patients with congenital glaucoma, the parents were first degree relatives (related to each other).

Following augmented trabeculectomy, eight eyes $(27.5 \%)$ required re-operation (one re-operation in five, and two to three re-operations in three). In three eyes, surgery was repeated during the first 3 months after trabeculectomy, in one eye after 6 months, and in four eyes, after 1 year. In one eye, a Molteno valve was inserted after two re-operations, and in two eyes $(6.8 \%)$, diode laser cyclophotocoagulation was performed after repeated trabeculectomies because of uncontrolled IOP. Of the remaining eyes, eight (27.5\%) received repeated subconjunctival injections of $5 \mathrm{mg} 5$-FU at 1-4 months after surgery because of the clinical appearance of the bleb and its vascularisation, and $13(44.8 \%)$ received additional antiglaucoma medications (one to four topical medications (mean 2.6); also including oral carbonic anhydrase inhibitor in one case), because of uncontrolled IOP.

Comparison of the preoperative and end of follow up indices (table 3 ) yielded a significant improvement in IOP,

Table 2 Diagnostic data in 17 children (29 eyes) with glaucoma

\begin{tabular}{ll}
\hline Diagnosis & \\
\hline Primary congenital glaucoma & 19 eyes $(65.5 \%)$ \\
Coloboma of iris and leucoma adherence & 1 eye $(3.4 \%)$ \\
Sturge-Weber syndrome & 1 eye $(3.4 \%)$ \\
$\begin{array}{l}\text { Congenital cataract } \\
\text { Posterior embryotoxon and iridocorneal }\end{array}$ & 4 eyes $(13.8 \%)$ \\
dysgenesis & 2 eyes $(6.9 \%)$ \\
Hypsarrhythmia (treated with steroids) & 2 eyes $(6.9 \%)$ \\
\hline
\end{tabular}

*An epileptic syndrome characterised by the triad of infantile spasms, hypsarrhythmia, and arrest of psychomotor development at seizure onset.

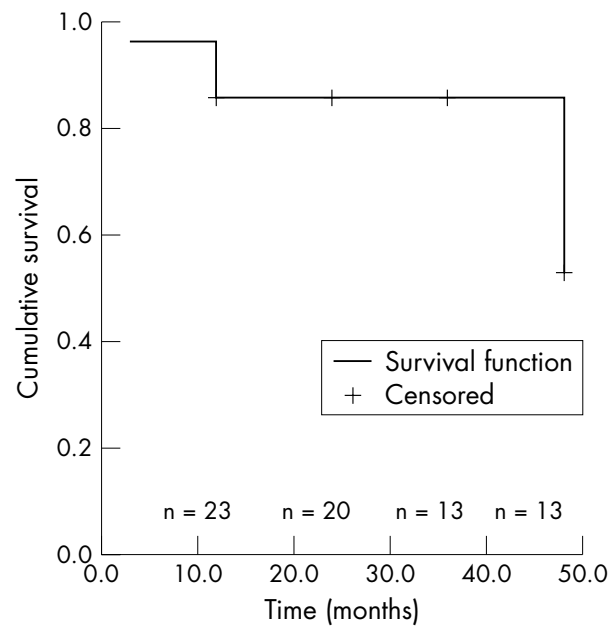

Figure 1 Kaplan-Meier survival analysis for eyes with intraocular pressure $<20 \mathrm{~mm} \mathrm{Hg}$.

from 21-60 mm $\mathrm{Hg}$ (mean 33.1 (10) $\mathrm{mm} \mathrm{Hg}$ ) before surgery to $6-26 \mathrm{~mm} \mathrm{Hg}$ (mean 17.1 (6) $\mathrm{mm} \mathrm{Hg}$ ) at the final examination $(\mathrm{p}<0.0001$, paired samples $t$ test $)$. In 22 eyes $(75.8 \%)$, the IOP was less than $20 \mathrm{~mm} \mathrm{Hg}$. Cup/disc ratio was $0.1-0.8$ (mean $0.42(0.26)$ ) before surgery and 0.1-1.0 (mean $0.511(0.27))$ at the final examination. The life table success rate for intraocular pressure control (fig 1) remained stable at $86 \%$ at 12, 24, 36 months and after 48 months decreased to $53 \%$. There was no significant difference in the life table results between primary and secondary glaucoma $(p=0.49)$ (fig 2). In five eyes (17\%), the cup/disc ratio progressed because of uncontrolled IOP. The difference between the initial and final ratios was not statistically significant ( $\mathrm{p}=0.45$, paired samples $t$ test). In one patient in whom both eyes were affected and IOP was not controlled, the cup/ disc ratio could not be determined owing to the presence of corneal oedema. Four patients were aphakic; in three pressure was controlled below $20 \mathrm{~mm} \mathrm{Hg}$ and because of their small number, we did not perform a separate data and statistical analysis of the group of aphakic patients.

Three of the patients were more than 1 year old (five eyes). In three of five eyes $(60 \%)$, pressure was $>20 \mathrm{~mm} \mathrm{Hg}$ at the end of follow up. Again, their small number precluded a

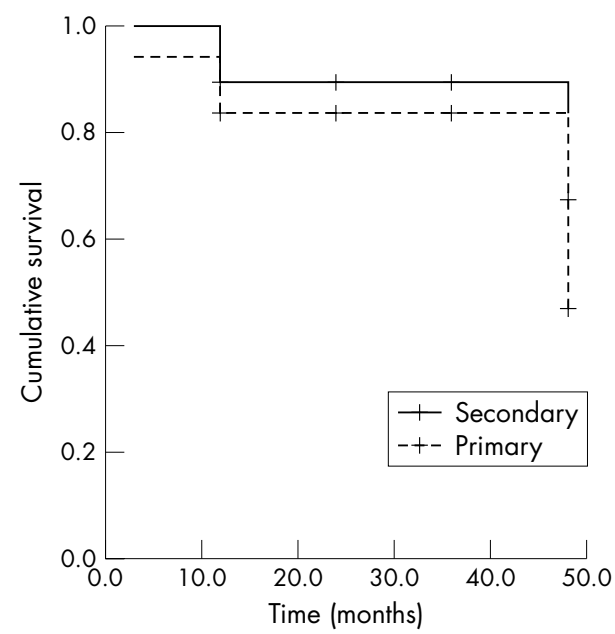

Figure 2 Kaplan-Meier survival analysis for eyes with intraocular pressure $<20 \mathrm{~mm} \mathrm{Hg}$. Comparison of patients with primary glaucoma and secondary glaucoma. 
separate statistical analysis. Their results were similar to the whole group of patients.

Cycloplegic refraction (in spherical equivalents (SE)) at the end of follow up ranged from +0.625 dioptres (D) to $+11.5 \mathrm{D}$ in 14 eyes $(48 \%)$ (mean +4.58 (3.73) D, hypermetropia range), and from $-0.5 \mathrm{D}$ to $-6.5 \mathrm{D}$ in seven eyes $(24 \%)$ (mean -3.28 (2.2) D, myopia range). Anisometropia $>2 \mathrm{D}$ was found in five patients. Visual acuity at the end of follow up ranged from 20/20 to no light perception. In 14 eyes $(48 \%)$, visual acuity was better than $20 / 120$. Two eyes $(6.5 \%)$ had no light perception at the end of follow up and two eyes $(6.5 \%)$ had corneal oedema that prevented reliable refraction assessment. In an additional four eyes (13\%), no refraction was documented in the medical file because of low compliance/lost to follow up. In the three patients who were more than 1 year old we were able to compare the visual acuity before surgery with end of follow up. In two of the eyes, visual acuity improved, from $20 / 200$ to $20 / 50$ in one, and from $20 / 200$ to $20 / 60$ in the other. Visual acuity was stable $(20 / 60)$ in one eye and decreased from 20/20 to $20 / 100$ in one. In one eye, visual acuity was 20/20 before surgery and decreased to no light perception because of acute endophthalmitis.

Late postoperative complications were noted in six eyes $(20.7 \%)$. One patient with bilateral congenital glaucoma and a positive family history had undergone several operations in another medical centre before being referred to ours. She was treated in our department for uncontrolled pressure in one eye with Ahmed valve implantation, but later developed acute fulminant endophthalmitis and ended with no light perception. Other complications (one eye each) included retinal detachment 1 year after surgery followed by phthisis bulbi, blebitis 3 years after surgery which was successfully

Table 3 Preoperative and end of follow up indices in 17 children (29 eyes) with glaucoma

\begin{tabular}{|c|c|}
\hline \multicolumn{2}{|l|}{ Indices, preoperative } \\
\hline Range & $21-60 \mathrm{~mm} \mathrm{Hg}$ \\
\hline Mean (SD) & $33.1(10) \mathrm{mm} \mathrm{Hg}$ \\
\hline \multicolumn{2}{|l|}{ Cup/disc ratio } \\
\hline Range & $0.1-0.8$ \\
\hline Mean (SD) & $0.42(0.26)$ \\
\hline \multicolumn{2}{|l|}{ Axial length (recorded in 11 eyes) } \\
\hline Range & $17-26 \mathrm{~mm}$ \\
\hline Mean (SD) & $22.38(2.43) \mathrm{mm}$ \\
\hline \multicolumn{2}{|l|}{ Corneal diameter (recorded in 23 eyes) } \\
\hline Range & $11-14.5 \mathrm{~mm}$ \\
\hline Mean (SD) & $12.7(1.2) \mathrm{mm}$ \\
\hline \multicolumn{2}{|l|}{ Postoperative treatment } \\
\hline Repeated surgery (1-3 procedures) & 7 eyes $(24.1 \%)$ \\
\hline Repeated 5-FU injection & 8 eyes $(27.5 \%)$ \\
\hline Diode laser & 2 eyes $(6.8 \%)$ \\
\hline Topical & 13 eyes $(44.8 \%)$ \\
\hline \multicolumn{2}{|l|}{ Indices, end of follow up } \\
\hline \multicolumn{2}{|l|}{ IOP } \\
\hline Range & 6-26 mm Hg \\
\hline Mean (SD) & $17.1(6.0) \mathrm{mm} \mathrm{Hg}$ \\
\hline \multicolumn{2}{|l|}{ Cup/disc ratio } \\
\hline Range & $0.1-1.0$ \\
\hline Mean (SD) & $0.511(0.27)$ \\
\hline \multicolumn{2}{|l|}{ Refraction (SE) } \\
\hline Hypermetropia & 14 eyes (48.2\%) \\
\hline Myopia & 7 eyes $(24.1 \%)$ \\
\hline Anisometriopia (>2D) & 5 patients $(29.4 \%)$ \\
\hline Visual acuity (20/20-no light perception) & 14 eyes $(>20 / 120)$ \\
\hline \multicolumn{2}{|l|}{ Complications } \\
\hline Endophthalmitis (after Ahmed shunt) & 1 eye $(3.4 \%)$ \\
\hline Retinal detachment + phthisis bulbi & 1 eye $(3.4 \%)$ \\
\hline Choroidal detachment & 1 eye $(3.4 \%)$ \\
\hline Blebitis & 1 eye $(3.4 \%)$ \\
\hline Corneal abscess in contact lens wearer & 1 eye $(3.4 \%)$ \\
\hline Thinning of bleb & 1 eye $(3.4 \%)$ \\
\hline
\end{tabular}

treated by re-operation; choroidal detachment caused by low IOP which resolved after intensive medical treatment; and corneal abscess in a patient wearing contact lenses after cataract extraction, which was treated with topical antibiotics. In addition, one patient showed antimetabolite induced thinning of the bleb.

\section{DISCUSSION}

The treatment of glaucoma in children poses a major challenge. The main goals of treatment are to control IOP and to preserve vision. The optimal surgical procedure remains controversial..$^{4-7}$ Traditionally, goniotomy and trabeculotomy are well accepted, with success rates of $80 \%$ or more. ${ }^{89}$ For good results, however, goniotomy requires a clear view of the trabecular meshwork, and may therefore be impossible to perform in the presence of opaque media caused by corneal oedema (about $50 \%$ of patients) ${ }^{1}$ or defects of the angle zone. ${ }^{2}$ Shaffer and Hoskins ${ }^{9}$ reported an overall success rate of $76.7 \%$ for goniotomy, which dropped to $30 \%$ when patients born with glaucoma and patients over the age of 24 months were analysed separately. They suggested that congenital glaucoma is associated with early arrest of embryonal development, resulting in a more severe anomaly, and that in patients older than 24 months, the trabecular meshwork responds less well because it is less cellular and contains more collagenous tissue. Other authors reported a success rate of only $33 \%$ for goniotomy as the primary operation. ${ }^{10}$

Unlike goniotomy, which requires a separate approach that is not familiar to most ophthalmologists, trabeculotomy is well studied and is currently the most common antiglaucoma procedure. ${ }^{11}$ However, the technique is highly demanding and requires previous experience and good anatomical landmarks to achieve predictable results. Furthermore, in some cases, Schlemm's canal may be difficult to canalise because of hypoplasia or abnormal anatomy. ${ }^{267}$ Previous studies of trabeculotomy reported success rates of $51 \%$ following 24 months of follow up. ${ }^{6}$

Several studies have found that the success of primary trabeculectomy in congenital glaucoma is at least equal to that of goniotomy and trabeculotomy. ${ }^{11}{ }^{13}$ Burke and Bowell ${ }^{14}$ reported a surgical success rate of $87 \%$ after a single procedure of trabeculectomy, which is higher than that for goniotomy and trabeculotomy. However, Beauchamp and Parks ${ }^{15}$ had a success rate of only $50 \%$ with trabeculectomy. It is controversial whether combined trabeculectomy and trabeculotomy is superior to trabeculectomy alone. A few authors reported better outcome with the use of both, ${ }^{211} 16$ whereas others noted no difference. ${ }^{17}{ }^{18}$ Song et al ${ }^{4}$ reported a $60 \%$ success rate for the first filtering operation; $20 \%$ of their patients required more than one surgical procedure. They found that the most important factor for success was the control of IOP, followed by the change in cup/disc ratio. Failure was related to older age ( $>1$ year), corneal diameter $>14 \mathrm{~mm}$, presence of secondary glaucoma, as well as previous surgery. In our series, there was no significant difference in outcome by patient age or primary as opposed to secondary glaucoma.

Filtering surgery is thought to be less successful in early childhood because of the thick and active Tenon's capsule, rapid healing response, and lower scleral rigidity associated with this age group. Furthermore, the conjunctival scarring from previous operations complicates repeated surgeries. As a result, the use of antimetabolites in children has been gaining popularity. Mandal et al ${ }^{19}$ reported a success rate of $94.7 \%$ with trabeculectomy supplemented with antimetabolites in older children (mean age 7.6 years), as opposed to Susanna et $a^{20}$ who had a success rate of only $59.5 \%$ in young children. The combination of trabeculectomy and trabeculotomy in 25 infants younger than 1 month (47 eyes) yielded good results 
in $71.7 \%$ after 3 years of follow up. ${ }^{21}$ The importance of age at surgery was supported by Al-Hazmi et al, ${ }^{22}$ who noted higher success rates for trabeculectomy as patient age increased, but also an increasing rate of antimetabolite induced complications. Freedman et $a l^{23}$ in a study of refractory paediatric glaucoma, found that the higher success rate for trabeculectomy with MMC and postoperative subconjunctival 5-FU was related to older patient age ( $>1$ years old) and to phakic eyes. Sidoti et $a^{24}$ reported their experience with augmented trabeculectomy with MMC in patients (mean age 6.4 years) with refractory glaucoma after failure with other modalities. They demonstrated survival rates of $82 \%$ at 12 months, $59 \%$ at 24 months, and $59 \%$ at 36 months.

A previous study by our group of trabeculectomy augmented with 5-FU and MMC versus 5-FU only yielded a higher success rate in the group treated with both antimetabolites. ${ }^{5}$ IOP was $<20 \mathrm{~mm} \mathrm{Hg}$ in $7 / 12$ eyes $(58.3 \%)$. Furthermore, at a mean follow up of 25 months IOP was controlled in seven of eight eyes treated with trabeculectomy augmented by MMC and 5-FU, but in none of the eyes $(0 / 4)$ treated with 5 -FU alone. In the present study, patients received MMC and 5-FU, in at least one of their trabeculectomy operations (in those with re-operations), so we could not perform a separate analysis of the different antimetabolites. This study reports the results of trabeculectomy with both antimetabolites in a larger group of patients with a long follow up period. Most of the patients were less than 1 year old yet did not present with a poor prognosis trend.

In comparison with others, ${ }^{17}{ }^{18}$ our results for augmented trabeculectomy with antimetabolites in 17 glaucomatous children were satisfactory. Most of the patients were males, and most (70\%) were affected bilaterally, in line with earlier reports in the literature. ${ }^{4}$ In $75.8 \%$ of the eyes $(22 / 29)$, IOP was controlled at $<20 \mathrm{~mm} \mathrm{Hg}$, life table success rate was $86 \%$ and remained stable for 36 months with a decrease to $53 \%$ after 48 months. The cup/disc ratio remained stable or improved. In the remaining $24.2 \%$ (7/29), the cup/disc ratio deteriorated. The decrease in IOP after surgery was statistically significant $(\mathrm{p}>0.0001)$, although the difference between the initial and final cup/disc ratios was not $(p=0.45)$. Most of the patients with uncontrolled IOP also showed deterioration in cup/disc ratio.

Our complication rate was low ( six eyes, $20.7 \%$ ). Beck et al ${ }^{25}$ reported choroidal detachment in $22 \%$ of patients, endophthalmitis in $8 \%$, and haemorrhagic choroidal detachment, retinal detachment, and vitreous haemorrhage in 3\%. Azuara-Blanco et $a^{26}{ }^{26}$ in a series of 21 patients, reported choroidal effusion in one patient, retinal detachment in one, and encapsulated bleb in one. Sidoti $e t a^{24}$ also reported a high rate of bleb related infections: $17 \%$ at mean follow up of 28 months. Blebitis occurred in in three of 29 patients, and endophthalmitis in two.

Interestingly, hypermetropia was the most common refractive error ( 14 eyes, $48.2 \%$ ), and myopia was found in only seven eyes $(24.1 \%)$; Mandal et $a^{21}$ found exactly the opposite relation. Anisometropia $>2 \mathrm{D}$ at the final examination was found in 10 eyes $(34.4 \%)$ in our series, compared to the $54.2 \%$ in the study of Mandal et al. ${ }^{21}$ In the remaining patients, refraction was not recorded. However, the present study is limited by the relatively small number of patients and the inclusion of patients with different types of glaucoma and of various ages (although most were between 1 month and 8 months old).

In conclusion, these data, together with the earlier study by our group, ${ }^{5}$ indicate that in selected paediatric cases of primary or secondary glaucoma in which visualisation of the trabecular meshwork is poor, trabeculectomy augmented with MMC and 5-FU is a good treatment option. In $75.8 \%$ of eyes, IOP was controlled at $<20 \mathrm{~mm} \mathrm{Hg}$ and the cup/disc ratio improved or showed no change, and in $50 \%$ of eyes visual acuity was better than 20/120. We attribute the low complication rate directly to the meticulous procedure and the appropriate use of antimetabolites. In our experience these patients can be very difficult to treat and sometimes have a very unpredictable follow up. Larger controlled studies are needed to determine the benefits of trabeculectomy alone versus combined trabeculectomy antimetabolites procedure.

\section{ACKNOWLEDGEMENTS}

We thank Gloria Ginzach and Hanni Penn for their editorial and secretarial assistance, and Dorit Karesh for the statistical analysis.

\section{Authors' affiliations}

R Ehrlich, M Snir, M Lusky, D Weinberger, R Friling, D D Gaton, Department of Ophthalmology, Rabin Medical Center, Beilinson Campus and Schneider Children's Medical Center of Israel, Petah Tiqva and Sackler Faculty of Medicine, Tel Aviv University, Tel Aviv, Israel

\section{REFERENCES}

1 De Luise VP, Anderson DR. Primary infantile glaucoma. Surv Ophthalmol 1983;28:1-19.

2 Elder MJ. Combined trabeculotomy-trabeculectomy compared with primary trabeculectomy for congenital glaucoma. Br J Ophthalmol 1994;78:745-8.

3 Meyer G, Schwenn O, Pfeiffer N, et al. Trabeculotomy in congenital glaucoma. Graefes Arch Clin Exp Ophthalmol 2000;238:207-13.

4 Song J, Stinnett SS, Whitson JT, et al. Ten-year surgical experience with childhood glaucoma. J Pediatr Ophthalmol Strabismus 2003:40:11-18.

5 Snir M, Lusky M, Shalev B, et al. Mitomycin C and 5-fluorouracil antimetabolite therapy for pediatric glaucoma filtration surgery. Ophthalmic Surg Lasers 2000;31:31-7.

6 Elder MJ. Congenital glaucoma in the West Bank and Gaza strip. Br J Ophthalmol 1993;77:413-6.

7 Harms H, Dannheim R. Epicritical consideration in 300 cases of trabeculotomy ab externo. Trans Ophthalmol Soc UK 1970;89:491-9.

8 Quigley HA. Childhood glaucoma: results with trabeculotomy and study of reversible cupping. Ophthalmology 1982;89:219-26.

9 Shaffer RN Hoskins HD. Montgomery lecture. Goniotomy in the treatment of isolated trabeculodysgenesis [primary congenital (infantile) developmental glaucoma]. Trans Ophthalmol Soc UK 1983;103:581-5.

10 McPherson SD Jr, Berry DP. Goniotomy vs external trabeculotomy for developmental glaucoma. Am J Ophthalmol 1983;95:427-31.

11 Mandal AK, Naduvilath TJ, Jayagandan A. Surgical results of combined trabeculotomy-trabeculectomy for developmental glaucoma. Ophthalmology 1998;105:974-82.

12 Debnath SC, Teichmann KD, Salamah K. Trabeculectomy versus trabeculotomy in congenital glaucoma. Br J Ophthalmol 1989;73:608-11.

13 Fulcher T, Chan J, Lanigan B, et al. Long-term follow up of primary trabeculectomy for infantile glaucoma. Br J Ophthalmol 1996;80:499-502.

14 Burke JP, Bowell R. Primary trabeculectomy in congenital glaucoma. Br J Ophthalmol 1989;73:186-90.

15 Beauchamp GR, Parks MM. Filtering surgery in children: barriers to success. Ophthalmology 1979;86:170-80.

16 Mullaney PB, Selleck C, Al-Awad A, et al. Combined trabeculotomy and trabeculectomy as an initial procedure in uncomplicated congenital glaucoma. Arch Ophthalmol 1999;117:457-60.

17 Beidner BZ, Rothkoff L. Combined trabeculotomy-trabeculectomy compared with primary trabeculotomy for congenital glaucoma. J Pediatr Ophthalmol Strabismus 1998;35:49-50.

18 Dietlein TS, Jacobi PC, Krieglstein GK. Prognosis of primary ab externo surgery for primary congenital glaucoma. Br J Ophthalmol 1999;83:317-22.

19 Mandal AK, Walton DS, John T, et al. Mitomycin C-augmented trabeculectomy in refractory congenital glaucoma. Ophthalmology 1997; 104:996-1001

20 Susanna R, Oltrogge EW, Carani JCE, et al. Mitomycin as adjunct chemotherapy with trabeculectomy in congenital and developmental glaucoma. J Glaucoma 1995;4:151-7.

21 Mandal AK, Gothwal VK, Bagga H, et al. Outcome of surgery on infants younger than 1 month with congenital glaucoma. Ophthalmology 2003:110:1909-15.

22 Al-Hazmi A, Zwaan J, Awad A, et al. Effectiveness and complications of mitomycin $C$ use during pediatric glaucoma surgery. Ophthalmology 1998; 105:1915-20.

23 Freedman SF, McCormick k, Cox TA. Mitomycin C-augumented trabeculectomy with postoperative wound modulation in pediatric glaucoma. J AAPOS 1999;3:117-24.

24 Sidoti PA, Belmonte SJ, Liebmann JM, et al. Trabeculectomy with mitomycin-C in the treatment of pediatric glaucomas. Ophthalmology 2000;107:422-9.

25 Beck AD, Wilson WR, Lynch MG, et al. Trabeculectomy with adjunctive mitomycin C in pediatric glaucoma. Am J Ophthalmol 1998;126:648-57.

26 Azuara-Blanco A, Wilson RP, Spaeth GL, et al. Filtration procedures supplemented with mitomycin $C$ in the management of childhood glaucoma. Br J Ophthalmol 1999;83:151-6. 